\title{
Perfeccionamiento del Programa de Control Sanitario Internacional en la provincia Holguín
}

\section{I mprovement of the International Health Program in Holguín province}

\section{Fernando González I sla}

Doctor en Medicina. Máster en Seguridad y Defensa Nacional. Especialista de I Grado en Medicina Interna. Dirección Provincial de Salud. Holguín, Cuba.

\begin{abstract}
RESUMEN
La Directiva No. 1 del Presidente del Consejo de Defensa Nacional para la Reducción de Desastres 2010, considera que existe una deficiente organización y cumplimiento de las medidas del Programa de Control Sanitario Internacional. El propósito es identificar las debilidades presentes en el Programa en la provincia Holguín y elaborar un plan de acción para elevar su efectividad. Se hizo revisión documental, aplicación de encuesta estructurada a 102 trabajadores vinculados con la ejecución del Programa, discusiones grupales, entrevistas y consulta a expertos. En todo los puertos se detectó la ausencia de incineradores para el tratamiento final de los desechos sólidos, en los centros provinciales, municipales y unidades de Higiene, Epidemiología y Microbiología, había poco dominio del porcentaje de muestras a enviar al laboratorio de referencia para el control y vigilancia del dengue y el paludismo y no dominaban los índices de vectores permisibles; en la atención primaria de salud, se encontró pobre capacitación sobre el Programa. Se elaboró el plan de acción. La implementación del plan de acción contribuye a la reducción de riesgos de epidemias y a la eliminación de las debilidades que limitan la actual efectividad del Programa de Control Sanitario Internacional en la provincia Holguín.
\end{abstract}

Palabras clave: Control Sanitario Internacional, provincia Holguín, Cuba. 


\title{
Revista Cubana de Salud Pública. 2011;37(3):259-275
}

\begin{abstract}
The Guideline No. 1 of the President of the National Council of Defense for Reduction of Disasters in 2010 states that the organization and the fulfilment of the measures included in the International Health Control program are unsatisfactory. The objective of this study is to identify the weaknesses of this program in Holguín province and to draw up an action plan to increase effectiveness. Methods Documentary review, structured survey administered to 102 people working in the program implementation, group discussions, interviews and expert advice were the methos used. It was detected that all the ports lacked burners for the final disposal of solid wastes; provincial, municipal and local Hygiene, Epidemiology and Microbiology centers showed poor knowledge about the percentage of samples to be sent to the reference laboratory for the control and surveillance of dengue and malaria, and about the allowable vector indexes; additionally, the primary health care staff was not duly trained in this program. The action plan was therefore prepared.

The implementation of the action program contributes to reducing epidemic risks and eliminating the weaknesses that restrict the effectiveness of the International Health Control at present in Holguín province.
\end{abstract}

Key words: International Health Control, Holguín province, Cuba.

\section{NTRODUCCI ÓN}

La historia del hombre ha estado marcada por la aparición de desastres sanitarios, grandes epidemias han afectado a la humanidad desde épocas remotas, un ejemplo típico fue la peste, conocida en China desde hace 3000 años y que en el siglo XIV ocasionó la muerte a un tercio de la población europea, la gripe desde el siglo XVI ha originado más de 31 pandemias y en 1918 mató a unos 20 millones de personas. ${ }^{1,2}$

La situación en las Américas es extremadamente seria, existe un número elevado de enfermedades infecciosas. Entre ellas merecen mención especial, el cólera que penetró a través de Perú, y produjo una epidemia en la región, excepto Uruguay en Sudamérica y los países del Caribe. El dengue se ha extendido por el área del Caribe y Centroamérica. En Venezuela, Colombia y Perú han aparecido nuevos serotipos de virus, lo que ha traído por consecuencia el aumento del dengue hemorrágico. La tuberculosis se ha incrementado principalmente en los países con situaciones económicas precarias y en las poblaciones marginales que se encuentran en la periferia de las grandes ciudades. La malaria es un serio problema de salud en la región, particularmente en Brasil, que presenta cepas de Plasmodium resistentes a las drogas. La infección por el $\mathrm{VIH} /$ sida considerada como la epidemia del siglo XX avanza en toda América con un número elevado de muertes y el incremento de personas seropositivas todos los años en proporciones catastróficas. ${ }^{3}$

Cuba no ha estado exenta de estas enfermedades. El número de pacientes con $\mathrm{VIH} /$ sida se mantiene en cifras bajas aunque los pacientes seropositivos se han incrementado de forma moderada. El dengue en su forma clásica y hemorrágica agredió a la población en los años 1981 y 1997. En la actualidad enfrentamos la epidemia de influenza A H1N1. 
En la provincia Holguín, en el año 1981 se presentó la epidemia conocida de dengue hemorrágico y en el municipio de Moa, en el año 2000 se notificó un caso de paludismo con transmisión local de la enfermedad al aparecer un total de 17 casos.

Para Cuba existe la posibilidad de graves epidemias si se tiene en cuenta la posición geográfica, la situación epidemiológica internacional de algunas enfermedades graves, la existencia de áreas vulnerables y brechas sanitarias, la posibilidad de una agresión biológica o la violación de la legislación sanitaria; elementos que pudieran facilitar la introducción y desarrollo de enfermedades epidémicas. A ello debemos añadir otros factores entre los que se destacan la proliferación de vectores incluido el mosquito Aedes aegypti, la amplia relación con países de Latinoamérica y África, el incremento del turismo y el creciente regreso de internacionalistas, ${ }^{1}$ (Directiva No.1 del Presidente del Consejo de Defensa Nacional para la reducción de desastres, 2010), así como el diagnóstico de casos importados de enfermos portadores de enfermedades trasmisibles, donde la provincia Holguín está entre las de mayor número de casos después de La Habana. ${ }^{4}$

Entre las medidas adoptadas en Cuba, con el objetivo primordial de evitar la introducción y propagación de un grupo de enfermedades transmisibles foráneas, se encuentran las regulaciones sanitarias de fronteras establecidas el 17 de enero de 1899. Desde 1902 aparecen las orientaciones del Dr. Carlos J. Finlay, en 1962 se estructura un Programa de Control Sanitario Internacional para puertos y aeropuertos y en 1980 se pone en vigor el primer Programa Nacional de Control Sanitario Internacional. ${ }^{5}$

Este importante Programa, en versión actualizada, está vigente desde marzo de 1999, por lo que se considera necesario evaluar su cumplimiento en la provincia Holguín, de forma tal que permita identificar las debilidades que pueden constituir brechas en la reducción de vulnerabilidades y riesgos en la prevención de epidemias.

Teniendo en cuenta esta situación de salud, es imprescindible saber cómo se puede lograr mayor efectividad en el Programa de Control Sanitario Internacional, para que contribuya a la reducción de riesgos de epidemias.

Las respuestas necesarias se pueden encontrar si se identifican las debilidades actuales en el Programa en la provincia Holguín y se elabora un plan de acción para elevar su efectividad, objetivos de esa investigación.

\section{MÉTODOS}

Se revisaron los documentos y regulaciones establecidas para el Control Sanitario Internacional, leyes, resoluciones, textos básicos y programas de esta disciplina; se diagnosticaron los principales elementos que caracterizan su estructura organizacional.

Se realizaron 102 encuestas a trabajadores vinculados a la ejecución del Programa de Control Sanitario Internacional en el año 2010, de ellos, 59 al personal que labora en la atención primaria de Salud, 24 al Centro Provincial de Higiene, Epidemiología y Microbiología (CPHE) y los centros y unidades municipales de Higiene, Epidemiología y Microbiología y 19 al personal que se desempeñan en las fronteras.

Por el riesgo que representa el municipio Holguín en la transmisión de enfermedades sujetas a control del Programa de Control Sanitario Internacional se aplicó la encuesta 
a otras personas, entre ellos, todos los Directores y Vicedirectores de las 10 áreas de salud de este municipio y en el área de Salud "Mario Gutiérrez Ardaya", por constituir la de mayor riesgo, se aplicó la encuesta a 21 de los 36 médicos vinculados con los consultorios médicos de la familia.

La investigación se estructuró en dos partes, en la primera se aborda la caracterización, antecedentes y la situación actual del Control Sanitario Internacional en la provincia Holguín y las principales debilidades que lo afectan. En la segunda, se incluye el plan de acción, validado por expertos, para perfeccionar este control.

\section{RESULTADOS}

\section{Caracterización del Control sanitario Internacional en la provincia Holguín}

Antecedentes y situación actual

Las enfermedades transmisibles (cualquier afección causada por un agente infeccioso o sus productos tóxicos) suelen clasificarse según diversos factores (agentes causales, puertas de entrada o salida, forma o vía de transmisión). Según las orientaciones del Programa de Control Sanitario Internacional, también se suelen agrupar desde el punto de vista práctico por los términos autóctono y exótico.

Las enfermedades autóctonas son todas aquellas en que se demuestre la transmisión en el país en las circunstancias actuales, por ejemplo la lepra, la tuberculosis, entre otras. Se consideran enfermedades exóticas aquellas que no han existido nunca en el país, o han sido eliminadas o erradicadas, y de las cuales no existe transmisión. Para Cuba, la malaria, la poliomielitis y otras. ${ }^{3}$

Dentro del ámbito científico-periodístico se ha extendido el uso de términos como enfermedades infecciosas emergentes y reemergentes, se consideran emergentes a aquellas infecciones nuevas aparecidas en una población dada en los últimos 20 años y reemergentes a aquellas enfermedades que existieron con anterioridad y se presumía que habían desaparecido o disminuido, y comienzan a elevar su incidencia o su alcance geográfico.

De manera intuitiva se puede reconocer que una enfermedad denominada exótica para un país, puede ser denominada como emergente y por otro lado, una enfermedad llamada autóctona para un país puede adquirir la categoría de reemergente. ${ }^{3}$

Las condiciones socioeconómicas y políticas de la globalización, con su enorme incremento del rápido contacto interhumano de muy diversas poblaciones de todas las latitudes, los cambios en el ambiente natural en distintos ecosistemas, la rapidez y eficiencia del transporte, el deterioro económico y la migración de grandes grupos poblacionales, entre otros, han influido en el surgimiento de nuevas enfermedades infecciosas antes desconocidas y al incremento inusitado de otras que se consideraban bajo control o en vías de desaparición. Estas enfermedades requieren de un sistema de vigilancia a nivel mundial, ante la posibilidad de provocar enfermedades epidémicas definidas como el número inusual de casos de una enfermedad que ocurre en un lugar y tiempo determinados. ${ }^{3}$

El Reglamento Sanitario Internacional (RSI) fue adoptado por primera vez en 1969, representa la primera iniciativa multilateral para prevenir la propagación internacional 


\section{Revista Cubana de Salud Pública. 2011;37(3):259-275}

de las enfermedades, en su inicio se estableció para vigilar, informar y controlar seis enfermedades transmisibles: cólera, peste, fiebre amarilla, viruela, fiebre recurrente y tifus. En 1973 se enmendó para incluir provisiones adicionales para el cólera y en el año 1981 se revisó una vez más para enfrentar la viruela, erradicada en 1979.

El 23 de mayo de 2005 la 58a Asamblea Mundial de la Salud (AMS) adoptó la nueva resolución del RSI (2005) revisado y actualizado, que entró en vigor el 15 de junio de 2007. EI RSI (2005) fortaleció varias resoluciones, entre ellas: WHA54.14 sobre la seguridad sanitaria mundial: alerta y respuesta ante epidemias y la WHA55.16 sobre respuesta mundial de salud pública a la aparición natural, la liberación accidental o el uso deliberado de agentes biológicos y químicos o de material radio nuclear que afecten a la salud. Este reglamento se aplica a las enfermedades con causas nuevas o desconocidas independientemente del origen o fuente, que signifiquen un daño importante a los seres humanos y motivado por el resurgimiento de algunas enfermedades infecciosas y el incremento del riesgo de propagación internacional, causado sobre todo por el crecimiento del transporte aéreo comercial. ${ }^{6}$

La finalidad y el alcance del RSI (2005) son prevenir la propagación internacional de enfermedades, proteger contra esa propagación, controlarla y darle una respuesta proporcionada y restringida a los riesgos para la salud, evitando al mismo tiempo las interferencias innecesarias con el tráfico y el comercio internacional. Este reglamento sirve de base jurídica a importantes documentos sanitarios relativos a los viajes y el transporte internacional, así como a la protección sanitaria de los usuarios de aeropuertos, puertos internacionales y pasos fronterizos terrestres. La Organización Mundial de la Salud (OMS) solicitó a los países comenzar a evaluar el sistema existente, mejorar la capacidad de detección, notificación, evaluación de riesgos, respuesta a los eventos de conformidad con el RSI (2005) y presentar informes sobre ellos.

En 1962 se estructura en Cuba un Programa de Control Sanitario Internacional para puertos y aeropuertos, y en 1980 se pone en vigor el primer Programa de Control Sanitario Internacional para preveer acciones a realizar por una parte de la red de servicios de salud y que sirvió de base para su modificación en 1985 cuando se extendió la vigilancia a toda la red de atención primaria y secundaria de salud con la incorporación de los médicos de familia; se permite así disponer de un sólido sistema de vigilancia a nivel de la población, centros de trabajo y educacionales y en el sistema turístico nacional, para detectar la ocurrencia de transmisión de enfermedades exóticas $u$ otros eventos de salud considerados extraños. La presencia de los Subdirectores de Higiene y Epidemiología en los policlínicos y la incorporación de la Unidad de Análisis y Tendencias en Salud (UATS) son nuevos elementos en el sistema que fortalecen la vigilancia. ${ }^{4}$

Por otra parte, la vigilancia se extendió a los Centros de Control de Viajeros en la fuente ubicados en los países con los cuales la colaboración es numerosa y el riesgo para la importación de enfermedades exóticas es mayor. Esta medida se considera una de las de mayor efectividad para evitar la introducción de tales enfermedades. ${ }^{7}$

Para hacer cumplir lo establecido en el Programa, el 6 de mayo de 1982 se dicta el Reglamento de las Disposiciones e Infracciones sobre el Control Sanitario Internacional donde se establecen las normas que deben regir, así como se determinan las acciones y omisiones no constitutivas de delitos y el procedimiento para la imposición y cobro de las multas correspondientes. ${ }^{8}$

El Ministerio de Salud Pública emite la Resolución 101 de fecha 2/6/2009 que establece nuevas indicaciones para el Control Sanitario Internacional a viajeros de Organismos de la Administración Central del Estado y de otras entidades nacionales 
(estas indicaciones no están incorporadas a la Directiva No. 1 del Presidente del Consejo de Defensa Nacional para Reducción de Desastres de 2010, ni a otros documentos que asegure su cumplimiento por los organismos en la provincia), además se diseñó el sistema de vigilancia para dicho control en la Misión Barrio Adentro en la República Bolivariana de Venezuela, como herramienta protectora de la salud de los colaboradores cubanos frente a enfermedades infecciosas y parasitarias, por lo que contribuye a la no introducción y propagación en Cuba de enfermedades exóticas por cubanos que regresen o por delegaciones de viajeros de otras nacionalidades procedentes de países endémicos e incorporados a los programas cubanos de colaboración. ${ }^{4,7}$

Se añade en las nuevas medidas que cada Dirección Provincial de Salud garantizará una cuarentena modificada a través de la creación del Centro de Chequeo a Colaboradores (aeropuerto u otra instalación), para el control de estos colaboradores, el mismo día de su arribo a la provincia (barrera de control de fronteras a nivel provincial), previo contacto con sus familiares.

\section{Consideraciones acerca de los elementos que caracterizan el Control Sanitario Internacional en la provincia de Holguín}

El Programa de Control Sanitario Internacional tiene como principal función reducir el riesgo de introducción de agentes biológicos, sus reservorios, vectores u hospederos intermediarios de las enfermedades sujetas a control, así como de otras enfermedades exóticas de interés sobre todo aquellas que no existen en el país. ${ }^{5}$

Un equipo de especialistas en los diferentes niveles del Sistema Provincial de Salud trabaja de forma coordinada con otros organismos e instituciones, incluye acciones en los puntos de entrada y salida de las fronteras (aeropuertos, puertos y marinas) y en especial, el trabajo en las áreas de salud y viviendas de los que arriban.

Se debe garantizar que las personas que salen del país tengan conocimiento en cuanto a los riesgos epidemiológicos a los que se pueden exponer y como minimizarlos. Por ejemplo, que viajen con los esquemas de vacunación completos y evaluados por una comisión médica, primordialmente quienes han sido designados a trabajar, estudiar o realicen viajes fuera de Cuba a países endémicos de enfermedades eliminadas en el país. Se considera endemia al número de casos habituales de una enfermedad, en un lugar determinado y durante un tiempo definido. ${ }^{3}$

Sobre los estudiantes y colaboradores en general debe mantenerse una vigilancia estricta de su estado de salud, con énfasis en las enfermedades infecciosas, para evitar que sean portadores al regreso, de padecimientos que puedan comprometer la salud de la población. Es lo que conocemos como el Control en la Fuente. Una vez en suelo patrio son controlados nuevamente en los puntos de entrada internacionales (aeropuertos, puertos y marinas) y se les garantiza un seguimiento estricto en su área de vivienda y trabajo durante 15 días en la búsqueda de sintomatología vinculada con procesos infecciosos para establecer el diagnóstico oportuno. ${ }^{7}$

Para lograr el cumplimiento del Programa es necesario ejecutar actividades tales como:

- Establecer las acciones higiénico-epidemiológicas que deben realizarse a nivel de fronteras (puertos y aeropuertos internacionales).

- Reordenar el sistema de vigilancia de viajeros internacionales acorde al riesgo personal y a la situación epidemiológica específica de los países de procedencia.

http://scielo.sld.cu 
- Definir las áreas geográficas de los países y los viajeros internacionales que deben ser considerados por el riesgo de convertirse en reservorios o portadores de enfermedades que no existen en el país.

- Establecer las acciones fundamentales para evitar la reintroducción del paludismo, dengue y cólera.

- Mantener un sistema de vigilancia sobre aquellos síndromes que pudieran ser expresión de la introducción de una enfermedad nueva emergente o reemergente.

- Tener estratificado a nivel local los riesgos fundamentales para la introducción de enfermedades exóticas para Cuba.

- Adiestrar y capacitar de forma continua al personal de salud sobre los cambios y modificaciones del Programa a través de la educación permanente.

- Incrementar las actividades de educación sanitaria individual y colectiva, en función de la situación epidemiológica particular de cada localidad.

- Lograr una participación activa de los organismos del estado en las actividades de protección y control al viajero. ${ }^{5}$

Como resultado de las medidas aplicadas, en la provincia Holguín no se han introducido enfermedades objeto del Reglamento Sanitario Internacional (fiebre amarilla, cólera, peste).

La introducción premeditada con carácter agresivo de vectores inexistentes o agentes infecciosos en Cuba, es otra poderosa razón que obliga a mantener y reforzar la vigilancia epidemiológica de enfermedades exóticas. La primera epidemia de dengue hemorrágico se presentó en 1981. En mayo de ese año se comenzaron a notificar algunos enfermos con síndrome febril compatible con el diagnóstico de dengue en el municipio Boyeros de La Habana. En ese mismo lugar, se comprobó retrospectivamente, la existencia de pacientes similares durante los meses anteriores. La enfermedad fue confirmada en La Habana, Cienfuegos, Camagüey y Holguín, en ese orden, siendo estas provincias las de mayor morbilidad. El acmé de la epidemia ocurrió a principios de julio de ese año; como resultado de las medidas higiénicosanitarias y de control vectorial intensivos, la epidemia disminuyó hasta darse por terminada el 10 de octubre del mismo año, fecha de la última notificación. ${ }^{4,9}$ (Martínez Torres E. Dengue [CD-ROM]. Ediciones Digitales).

Entre los años 2004 y 2008, la introducción de casos importados de dengue en la provincia de Holguín alcanza la cifra de 17, en su mayoría colaboradores procedentes de áreas endémicas, principalmente de Venezuela, lo que refuerza la necesidad de extremar las medidas de control relacionadas con esta enfermedad. No se han producido brotes secundarios a estos casos importados, lo que constituye un excelente resultado en la reducción de riesgos de enfermedades epidémicas.

Dentro de las enfermedades sujetas a vigilancia epidemiológica internacional, el paludismo o malaria ha sido la que mayor riesgo de introducción ha presentado en los últimos años y contra lo cual se han tenido que adoptar medidas de control especiales. Entre los años 2004 y 2008 se notificaron en Holguín nueve casos importados, y no han ocurrido brotes de transmisión local. La prevención de la introducción de esta enfermedad ha sido efectiva, lo que demuestra las potencialidades del Control Sanitario Internacional, cuando se realizan las acciones previstas con calidad. 


\section{Revista Cubana de Salud Pública. 2011;37(3):259-275}

En la provincia el Programa está estructurado en todos los niveles de atención del Sistema Nacional de Salud, que incluye a los consultorios médicos de la familia como eslabón imprescindible para su éxito.

La vigilancia en frontera se lleva a cabo a través del Aeropuerto Internacional Frank País García, los puertos de Moa, Nicaro, Felton, Antilla y Rafael Freyre y la Marina de Vita en el municipio Rafael Freyre.

En cada uno de los eslabones del sistema de salud se ejecutan acciones específicas. A nivel de puertos y aeropuertos se debe realizar el despacho sanitario del $100 \%$ de las naves y aeronaves procedentes del extranjero, para lo cual las autoridades del Control Sanitario Internacional exigirán a los organismos estatales correspondientes la información oportuna y detallada del arribo de buques y aeronaves internacionales.

Los responsables del Control Sanitario Internacional otorgarán a petición de las autoridades del buque o sus representantes la libre plática, por radio cuando la información facilitada por estos antes de su arribo y la situación epidemiológica existente en los países de procedencia así lo permitan y la misma se haya realizado en un tiempo comprendido entre 24 y $48 \mathrm{~h}$ antes del arribo del buque.

Cuando la información solicitada para otorgar la libre plática, no cumpla los requisitos de calidad y oportunidad, esta será entregada por el médico de cuarentena durante su visita al buque. La autoridad de Control Sanitario Internacional será la primera en abordar al buque para realizar la inspección sanitaria (médico de cuarentena y técnico de vectores).

Una vez realizada la inspección documental, la autoridad de Control Sanitario Internacional procederá a la inspección sanitaria del buque o aeronave, revisando los compartimentos (total de pasajeros y equipajes, camarotes, cocina, servicios sanitarios, entre otros), supervisando la higiene personal, recolección de desechos sólidos y líquidos, desperdicios y restos de alimentos.

Si en la inspección se detectaran violaciones del RSI o de las leyes sanitarias vigentes, en ese momento se notificará a la persona o entidad causante de la infracción cometida, para la aplicación de la multa correspondiente.

Las unidades de Control Sanitario Internacional de puertos y aeropuertos ejecutarán en todo el perímetro de las áreas portuarias y aeroportuarias $(400 \mathrm{~m})$, las actividades correspondientes a los programas de salud ambiental.

En los puertos y aeropuertos internacionales se exigirá el perfecto funcionamiento del incinerador para el tratamiento (destrucción) de los residuales sólidos generados en aeronaves y buques, así como su correcta disposición y transportación hasta el incinerador. Se exigirá además el perfecto funcionamiento de la fosa internacional donde se disponen los residuales líquidos de las aeronaves para su tratamiento y posterior disposición al sistema local de alcantarillado. En los aeropuertos donde no exista fosa internacional, los aviones no podrán descargar sus residuales, siendo muy rigurosa la autoridad sanitaria en velar en que no se derramen estos residuales sobre la losa del aeropuerto.

Las embarcaciones de todo tipo que se encuentren atracadas o fondeadas en los puertos, bahías o aguas de jurisdicción cubana, no podrán verter los residuales líquidos, por lo que se les clausuran las salidas de sus tanques sépticos por las autoridades de seguridad marítima al momento de su incorporación al territorio nacional. Las embarcaciones que disponen de sistema de reciclaje de los residuales 


\section{Revista Cubana de Salud Pública. 2011;37(3):259-275}

líquidos no representan riesgo, pero se debe controlar que estos sistemas funcionen correctamente.

A los viajeros internacionales que arriben por vía marítima, el médico de cuarentena examinará a cualquier tipo de enfermo que hubiese a bordo y viabilizará su ingreso hospitalario o aislamiento si sospecha de la presencia de alguna enfermedad transmisible, lo que notificará de forma oportuna y completa al CPHE y de este a la UATS Nacional, estableciendo las medidas de control requeridas para evitar su propagación.

Los viajeros sometidos a vigilancia epidemiológica seguirán los mismos pasos que los descritos para los viajeros que arriben por vía aérea. Cuando existan antecedentes o sospecha de enfermedades transmisibles a bordo, la autoridad sanitaria prohibirá el ascenso o descenso del buque de cualquier persona hasta tanto no se haya terminado su inspección, delimitando el riesgo y adoptando las medidas pertinentes. Se exceptúan de esta disposición a los prácticos de los puertos.

En el CPHE se obtendrá a través de los organismos estatales la información acerca de la salida de viajeros internacionales, y le brindará toda la información necesaria para la prevención de enfermedades en dependencia del país donde se realice el intercambio. Recibirán desde los aeropuertos (vía télex, correo electrónico o teléfono) las listas de viajeros cubanos sometidos a vigilancia epidemiológica y a los que se les entregó tarjeta de advertencia. En un plazo no mayor de $24 \mathrm{~h}$ informará a los municipios la relación nominal de los viajeros recibidos. El departamento de epidemiología, a través del J efe del Programa de Control Sanitario Internacional, despachará semanalmente con los municipios la situación de cada viajero sometido a vigilancia y procederá a la localización de aquellos que por alguna razón se informen como ilocalizables. De igual forma, hará una evaluación mensual de la marcha de este Programa la que remitirá a la Dirección Nacional de Epidemiología y retroalimentará al Instituto de Medicina Tropical "Pedro Kouri" (IPK) sobre el control de los viajeros.

Los 14 municipios de la provincia Holguín cuentan con unidades y centros municipales de higiene y epidemiología, que reciben la listas de los viajeros de parte de la provincia, y lo hacen llegar a las áreas de salud en un plazo no mayor de $24 \mathrm{~h}$. Semanalmente el epidemiólogo municipal a cargo del Programa, despachará con los Subdirectores de Higiene y Epidemiología de las áreas de salud los resultados de la vigilancia y las dificultades que se presenten en este sentido. En el control de las estrategias del área de higiene y epidemiología, se realizará una evaluación quincenal de la marcha de este Programa, y se brindará información semanalmente al J efe del Programa de la provincia de los resultados de la vigilancia.

En los 46 policlínicos de la provincia se hará entrega de la relación de los viajeros internacionales a los consultorios médicos de la familia en las primeras $24 \mathrm{~h}$ de haberlos recibidos, para lo cual se utilizará la vía del Sistema de Información, Grupos Básicos de Trabajo o personal de la subdirección de higiene y epidemiología del policlínico; en dependencia de las características de cada área, conocerá en un plazo de $24 \mathrm{~h}$ la conducta seguida por el médico en el control del viajero, informará a la dirección del municipio de la conducta seguida con los viajeros y procederá a la localización de aquellos que se den como ilocalizables por el médico de la familia y evaluará semanalmente la marcha de este Programa con la participación de los Grupos Básicos de Trabajo y otras áreas relacionadas.

En los 25 hoteles y villas turísticas laboran 36 médicos y 28 enfermeras, que conocen diariamente el personal hospedado y los países de procedencia, se mantienen informado de la situación internacional para poder conocer los riesgos a que están expuestos y poder orientar la vigilancia en dependencia de estos. En caso de 


\section{Revista Cubana de Salud Pública. 2011;37(3):259-275}

detectarse algún huésped con sintomatología de algunas de las enfermedades de interés epidemiológico, procederá al ingreso hospitalario en la unidad que corresponda según territorio e informará de inmediato al nivel correspondiente de la situación existente. ${ }^{5}$

En el año 2009 arribaron a la provincia por el aeropuerto internacional un total de 1 799 vuelos, con 226420 pasajeros que entraron, y 234591 que salieron, lo que significa un incremento de 50543 en relación con el año 2008. Los países con mayor cantidad de turistas fueron: Canadá, Alemania, Holanda, Inglaterra e I talia.

A través de los puertos llegaron 91 buques, con 1785 tripulantes extranjeros procedentes de España (246), Trinidad y Tobago (214), Alemania (196) y Canadá (184).

Con destino a la provincia, se recibieron 176027 viajeros, de los cuales 5445 procedieron de áreas endémicas de enfermedades sujetas a vigilancia, de ellos 3889 son colaboradores cubanos y 1556 extranjeros ( 818 estudiantes). Los países con mayor número de viajeros son: Venezuela con 3644 y Guatemala con 89. Los sectores a los que pertenecen el mayor número son: salud publica con 3432 y el I NDER con 316. En relación con el año 2008 la cantidad de viajeros es similar ( 175 877).

La vigilancia epidemiológica a la población de la provincia identificó 777072 personas como de riesgo (expuesta) y con síndromes febriles inespecíficos 367 . Se realizaron 11946 gotas gruesas en la vigilancia de paludismo y 509 monosueros para dengue.

Durante el año 2009 se realizó el diagnóstico de un paciente con paludismo importado y 9 de dengue, no se generaron casos secundarios a partir de estos. El paciente portador de paludismo procedía de Angola (cubano invitado por familiares) y los de dengue correspondieron a siete colaboradores procedentes de Venezuela, de ellos cinco del sector de la salud y dos del INDER, una estudiante boliviana y una persona procedente de Haití.

Entre las principales actividades de control realizadas dentro del Control Sanitario Internacional en los últimos meses, está lo relacionado con el enfrentamiento a la pandemia de influenza $A(H 1 N 1)$ del 2009, que ha puesto de manifiesto la respuesta inmediata ante una enfermedad emergente, causada por una variante del virus detectado en México el 18 de marzo de 2009, el 29 de abril la OMS elevó el nivel de alerta por pandemia de influenza a la fase $5 .{ }^{10}$

Hasta el 7 de mayo del año 2010 se han producido un total de 18001 fallecidos, en las Américas ( 8 357),

Europa (4 860), Pacífico occidental ( 1810 ), Mediterráneo oriental ( 1 019), Sudeste asiático ( 1787 ) y África (168). Por países Estados Unidos aporta el mayor número de defunciones con 2 718, Brasil 2 101, México 1182 y Argentina 626. En Cuba han fallecido 63. En la provincia Holguín se notificaron 109 casos con dos fallecidos confirmados.

Los grupos más vulnerables para la Influenza A H1N1 son las embarazadas, recién nacidos, niños menores de 5 años, pacientes con antecedentes de asma, diabetes, inmunosupresión, enfermedades respiratorias y cardiovasculares. Los casos más severos y las muertes han ocurrido en adultos menores de 50 años y la inmensa mayoría de los pacientes han teniendo síntomas moderados. 


\section{Principales debilidades que afectan el Control Sanitario I nternacional en la provincia Holguín}

La reducción de riesgos asociados a epidemias es una obligación estatal, con la participación de diferentes organismos y el papel rector de Salud Pública, concebido el riesgo como la posibilidad y proximidad de que suceda un daño; es función de amenaza y vulnerabilidad. El CSI constituye un eslabón de gran importancia en el logro de ese objetivo, por lo que se dirigen importantes esfuerzos para lograrlo, y se incluyen acciones de control por el nivel nacional y provincial. ${ }^{7,11}$

En entrevista concedida por el J efe del Programa de Control Sanitario Internacional en la provincia señala como principales dificultades del Programa, los siguientes:

- No acudir dentro de las primeras $72 \mathrm{~h}$ al médico de asistencia.

- Indisciplinas en el control de estudiantes y colaboradores procedentes de otros países que arriban a Cuba y les falta el examen de control de salud o no han declarado el padecimiento de alguna enfermedad.

- Baja percepción de riesgo en la comunidad que posibilita la proliferación de vectores.

- Falta de responsabilidad de los representantes de los organismos en el control de sus viajeros.

- Brechas aún existentes en el Sistema Nacional de Salud en las pesquisas activas de los viajeros

- La responsabilidad ciudadana no está presente en el cumplimiento de los requisitos establecidos.

Todos estos problemas pudieran ser sancionables con multas por violación de la legalidad, al ser un posible trasmisor de epidemias.

A todas luces resulta prácticamente imposible un riguroso control de las enfermedades infecciosas sin la participación de todos. Algunos de los brotes, fundamentalmente de dengue y conjuntivitis notificados en el país, están relacionados directamente con viajeros que han ocultado su verdadero estado de salud, lo que ha traído como consecuencia la transmisión local de esas enfermedades. ${ }^{12}$

En las tres instancias siguientes se identificaron como principales debilidades las siguientes:

\section{En fronteras}

- La ausencia de incineradores en los puertos para el tratamiento final de los desechos sólidos internacionales.

- Desconocimiento de los principales documentos que se les debe exigir, tanto a los buques como a las aeronaves desde el punto de vista sanitario $(84,2 \%$ del personal demostró dominio).

- Poco dominio de los índices de vectores en frontera, solo el 63,1 \% domina adecuadamente los valores permisibles.

http://scielo.sld.cu 


\section{En el Centro Provincial de Higiene, Epidemiología y Microbiología y centros y unidades municipales de Higiene, Epidemiología y Microbiología}

- El 83,3 \% de los encuestados demostró saber que la vigilancia epidemiológica de los viajeros debe realizarse durante los primeros 15 días a partir de su llegada.

- No hay un óptimo dominio de los criterios de evaluación de la vigilancia de paludismo según estratificación de riesgo, solo el 75,0 \% dominaba la forma en que se debe vigilar esta entidad.

- Poco dominio del porcentaje de muestras a enviar al laboratorio de referencia para el control y vigilancia de dengue $(66,6 \%)$ y paludismo $(75,0 \%)$.

\section{En la atención primaria de salud}

- Insuficiente capacitación en el Programa de Control Sanitario Internacional, solo el $66,1 \%$ de los encuestados refirieron haber recibido adiestramiento.

- El 74,5\% planteó recibir diariamente las listas sobre el control y vigilancia de los viajeros procedentes de áreas endémicas.

- El 10,1 \% dominaba los criterios de evaluación de la vigilancia de paludismo según estratificación de riesgo.

- El 76,2 \% de los encuestados demostró conocer a quiénes se le debe aplicar la profilaxis y tratamiento radical antipalúdico.

- Poco dominio del porcentaje de muestras a enviar a laboratorio de referencia para el control y vigilancia de paludismo $(47,4 \%)$.

- Desconocimiento de los principales indicadores de vigilancia de dengue, solo el 74,5 $\%$ domina la conducta a seguir ante un síndrome febril inespecífico, y el 84,7 \% la situación de la focalidad en el territorio.

- Solo el 86,4\% de los encuestados domina los elementos a tener en cuenta en la preparación integral del colaborador antes de su salida del país.

Al analizar de conjunto los tres niveles o instancias donde se ejecuta el Programa de Control Sanitario Internacional, las mayores fortalezas se encuentran en el personal que se desempeña en los puntos de frontera, mientras que los de la atención primaria de salud necesitan de un proceso sostenido de capacitación y control. En los que laboran en el Centro Provincial de Higiene, Epidemiología y Microbiología y en los centros y unidades municipales correspondientes, deben incrementar sus conocimientos en algunos indicadores del Programa, para que puedan desempeñar adecuadamente sus funciones de asesoría y control.

\section{PLAN DE ACCIÓN PARA PERFECCIONAR EL CONTROL SANITARIO I NTERNACI ONAL EN LA PROVI NCI A DE HOLGUÍ N}

La seguridad nacional en materia de salud pública es un desafío en el panorama del mundo actual, compartir la vulnerabilidad implica compartir también la responsabilidad, por lo que se requiere fortalecer los sistemas de prevención y respuesta oportuna a los riesgos de enfermedades que pueden provocar epidemias. ${ }^{6}$ 
Se propone a partir de las debilidades encontradas, un plan de acción (anexo) para mejorar la efectividad del Control Sanitario Internacional; concebida esta como el grado en que determinadas acciones consiguen producir una mejoría en el grado de salud del paciente o de la población, en condiciones reales de aplicación, y contribuir a la reducción de riesgos de desastre sanitario (epidemias). Para cada una de las principales dificultades se asigna un objetivo concreto cuya consecución contribuye al perfeccionamiento del Programa. ${ }^{3}$

De los nueve expertos consultados, siete validaron sin reservas el plan de acción propuesto, lo que significa que el $77,8 \%$ se identificó totalmente con su contenido y dos de ellos suscribieron la mayor parte, teniendo algunas sugerencias, relacionadas con la incorporación de acciones dirigidas a actores externos.

Se puede concluir que el Control Sanitario Internacional ha contribuido de forma significativa a la reducción de riesgos de graves epidemias. Las insuficiencias detectadas en el cumplimiento del Programa de Control Sanitario Internacional, si bien no son la causa de aparición de brotes epidémicos en la provincia, constituyen vulnerabilidades que entrañan un riesgo potencial y deben ser reducidos. El plan de acción responde a las principales debilidades identificadas y se elaboró con un enfoque sistémico.

Se recomienda proponer al J efe del Estado Mayor Nacional de la Defensa Civil incluir en las indicaciones metodológicas para el cumplimiento de la Directiva No. 1 del Presidente del Consejo de Defensa Nacional para la reducción de desastres, las medidas a cumplir por los diferentes Organismos de la Administración Central del Estado relacionados con la colaboración internacional dentro del Programa de Control Sanitario Internacional. Sugerir al Ministerio de Salud Pública la actualización del Programa de Control Sanitario Internacional a partir de que el vigente, es de marzo de 1999, y no se ajusta a las nuevas condiciones y problemas de salud. Proponer al Ministerio de Salud Pública incorporar a los Programas de estudio de pregrado y posgrado los contenidos del Programa de Control Sanitario Internacional. Presentar en la provincia los resultados del trabajo. Proponer que se diseñe un plan provincial de capacitación por categoría ocupacional del personal de salud involucrado en la vigilancia del Control Sanitario Internacional, con periodicidad por lo menos anual y tenga en consideración las particularidades de los trabajadores según las necesidades de aprendizaje identificadas. Dar continuidad a la investigación, en lo relacionado con el cumplimiento de las funciones de los organismos vinculados con el Programa y completar la validación de los resultados.

\section{Anexo}

\section{Objetivo 1}

Garantizar que a nivel de las diferentes instituciones y puntos de frontera se dispongan de las condiciones estructurales necesarias para el cumplimiento del Programa.

Criterio de medida:

Se cuenta con incineradores en los puertos de Moa, Nicaro, Felton, Antilla y Rafael Freyre. 


\section{Revista Cubana de Salud Pública. 2011;37(3):259-275}

Acciones:

- Exigir a los organismos responsables la construcción de los incineradores en los puertos. Ejecutan las tareas los Directores de las unidades municipales de higiene y epidemiología (UMH ) y centros municipales de higiene y epidemiología (CMHE), Técnicos de la Inspección Sanitaria Estatal (ISE). Son responsables el Director Provincial de Salud Pública y el Director CPHE. Cumplen las tareas en septiembre de 2010.

- Aplicar la legislación establecida ante el incumplimiento de lo previsto sobre la existencia de incineradores. Ejecutan las tareas los técnicos de la ISE. Son responsables el director Provincial de Salud Pública y el director CPHE. Cumplen las tareas en diciembre de 2010.

- Informar periódicamente al Partido y al Gobierno en la Provincia y al MINSAP el estado de los incineradores. Ejecuta el Director CPHE. Es responsable el Director Provincial de Salud Pública. Cumple la tarea en septiembre de 2010 y de enero a julio de 2011.

\section{Objetivo 2}

Mejorar el nivel de preparación del personal de salud sobre el Programa de Control Sanitario internacional.

Criterio de medida:

El personal de salud se encuentra debidamente preparado para el cumplimiento del Programa de Control Sanitario Internacional.

Acciones:

- Facilitar los programas y materiales de consulta actualizados para la autopreparación de los involucrados en el Control Sanitario Internacional. Ejecutan las tareas el Vicedirector de Epidemiología, el jefe del departamento de Control Sanitario Internacional del CPHE y el responsable del Centro de Información de Ciencias Médicas. Son responsables el Director de CPHE y el Rector de la Universidad de Ciencias Médicas. Cumplen la tarea en octubre de 2010.

- Diseñar actividades capacitantes de posgrado en sus diferentes modalidades. Ejecutan los especialistas de Higiene y Epidemiología, metodólogos de la Universidad de Ciencias Médicas. Son responsables el Director de CPHE y el Rector de la Universidad de Ciencias Médicas. Cumplen la tarea en noviembre de 2010.

- Realizar evaluación periódica de competencia y desempeño a los involucrados en el Control Sanitario Internacional. Ejecuta el Grupo Asesor de Control Sanitario Internacional de la provincia. Son responsables el Director de CPHE y el jefe del departamento de Control Sanitario Internacional del CPHE. En septiembre de 2010 deben tener el diseño del sistema a utilizar en esa fecha. septiembre de 2010.

- Capacitar al personal asignado para el manejo de la información. Ejecuta el J efe del Departamento de Control Sanitario Internacional. Son responsables el Director de CPHE y el Rector de la Universidad de Ciencias Médicas. Cumplen la tarea en noviembre de 2010.

\section{Objetivo 3}

Lograr resultados óptimos de los indicadores de la vigilancia epidemiológica concebidos en el Programa de Control Sanitario Internacional.

http://scielo.sld.cu 


\section{Revista Cubana de Salud Pública. 2011;37(3):259-275}

Criterio de medida:

Se cumplen los parámetros establecidos en el Programa de Control Sanitario Internacional para la vigilancia epidemiológica de los viajeros extranjeros que arriban a la provincia.

Acciones:

- Sistematizar el análisis epidemiológico de riesgos y eventos en los diferentes niveles de actuación. Ejecutan los responsables del Control Sanitario Internacional según nivel de actuación. Es responsable el J efe Departamento de Control Sanitario Internacional del CPHE. Cumplen las tareas, diario en las áreas de Salud, semanal en el nivel municipal y provincial.

- Verificar el cumplimiento de la vigilancia por personal especializado en las áreas de mayor riesgo. Ejecutan los profesionales y técnicos seleccionados. Es responsable el J efe Departamento de Control Sanitario Internacional en municipios y provincia. El cumplimiento es semanal en las áreas seleccionadas.

- Evaluar por los principales cuadros el cumplimiento de los indicadores de la vigilancia epidemiológica. Ejecutan los Directores de áreas de salud, de UMHE y de CMHE. Son responsables el Director Provincial de Salud Pública y el Director CPHE. El cumplimiento es semanal en el nivel municipal y mensual en el provincial.

\section{Objetivo 4}

Mejorar la preparación de las personas que participan en la colaboración internacional, estudiantes extranjeros y funcionarios de los organismos, en función de lograr una participación más activa en la prevención de enfermedades sujetas a Control Sanitario Internacional.

Criterio de medida:

Las personas involucradas en la colaboración internacional participan activamente en la prevención de enfermedades sujetas a Control Sanitario Internacional.

\section{Acciones:}

- Implementar los planes de preparación en todas las entidades involucradas en la colaboración, según el nivel de riesgo. Ejecutan los Directores de las entidades involucradas en la colaboración internacional y los responsables de la colaboración en los organismos. Son responsables los Directores de Organismos en la Provincia, Director CPHE, Universidad Medica. Cumplen la tarea en septiembre de 2010.

- Evaluar en el personal que participa en la colaboración y en los estudiantes el nivel de conocimientos sobre las enfermedades sujetas a Control Sanitario Internacional. Ejecutan los Vicedirectores de Higiene y Epidemiología y Jefes de Grupos Básicos de Trabajo de las áreas de salud. Son responsables los Directores Municipales de Salud y de áreas de salud. Cumplen la tarea antes de su incorporación a la colaboración.

\section{Objetivo 5}

Fortalecer los mecanismos de control del Programa de Control Sanitario Internacional.

Criterio de medida:

Se cumple lo normado en el Programa de Control Sanitario Internacional.

Acciones:

- Crear equipo provincial y municipal de fiscalización para el cumplimiento de las 
actividades en Control Sanitario Internacional. Ejecutan el J efe de Departamento de Control Sanitario Internacional del CPHE, los CMHE y UMHE. Son responsables el Director Provincial de Salud y Director del CPHE. Cumplen la tarea eb septiembre de 2010.

- Crear instrumentos y comisión provincial para la acreditación de todas las entidades involucradas en el Control Sanitario Internacional. Ejecuta el J efe de Departamento de Control sanitario Internacional del CPHE. Son responsables el Director Provincial de Salud y el Director del CPHE. Cumplen en octubre de 2010.

- Implementar sistema informatizado para la recolección, procesamiento y envió de la información. Ejecuta el Jefe de Departamento de Informática de la Dirección Provincial de Salud y el J efe de Departamento de control sanitario Internacional del CPHE. Responden por la tarea el Director Provincial de Salud y el Director del CPHE. Cumplen en noviembre de 2010.

- Aplicar el reglamento sobre las disposiciones e infracciones del control sanitario Internacional. Ejecutan los técnicos de ISE. Son responsables los Directores de las UMHE, los CMHE y el Director de CPHE. El cumplimiento es diario según la existencia de violaciones.

- Evaluar la aplicación del reglamento sobre las disposiciones e infracciones sobre el Control Sanitario Internacional. Ejecutan los responsables del Control sanitario Internacional según nivel de actuación. Son responsables los Directores de las UMHE, CMHE y el Director de CPHE. El cumplimiento es semanal en la evaluación del Programa y mensual en la evaluación del Programa.

\section{REFERENCI AS BI BLI OGRÁFICAS}

1. Navarro Machado VR. Situaciones de desastres. Manual para la preparación comunitaria. Cienfuegos: Ediciones Damují 2007; 2009.

2. Las primeras epidemias de la historia [Internet]. [citado 3 Abr 2010].Disponible en: http://www.sld.cu/galerias/pdf/sitios/bmn/las_primeras_epidemias_de_la_historia.pdf

3. Toledo Curbelo GJ, Rodríguez Hernández P, Reyes Sigarreta M, Cruz Acosta A, Caraballoso Hernández M, Sánchez Santos L, et al. Fundamentos de Salud Pública. La Habana: Editorial Ciencias Médicas; 2005.

4. Ávalos Quesada RM. Evaluación de la vigilancia del Control Sanitario Internacional en Plaza de la Revolución, año 2008 [tesis]. La Habana: Instituto Nacional de Higiene Epidemiología y Microbiología (INHEM)); 2009.

5. Ministerio de Salud Pública de Cuba. Actualizaciones del Programa Nacional de Control Sanitario Internacional [Internet]. La Habana: MI NSAP; 1998 [citado 3 Abr 2010]. Disponible en: http://files.sld.cu/sida/files/2010/11/programa-controlsanitario-internacional.pdf

6. Organización Mundial de la Salud. Reglamento Sanitario internacional [Internet]. Ginebra: OMS; 2005 [citado 6 Mar 2010]. Disponible en:

http://www. who.int/csr/ihr/es/es/index.html

http://scielo.sld.cu 
7. Ramírez Rodríguez M, Fariñas Reinoso AT, Alfonso Berrios L. Diseño del sistema de vigilancia para el control sanitario internacional (control en la fuente). Reporte Técnico de Vigilancia [Internet]. República Bolivariana de Venezuela: Misión Barrio Adentro; 2007 [citado 3 Abr 2010]; 12(4). Disponible en:

http://www.sld.cu/galerias/pdf/sitios/vigilancia / ramirezmilvia.pdf

8. Gaceta Oficial de la República de Cuba [Internet]. Edición Ordinaria. Reglamento de las Disposiciones e infracciones sobre Control Sanitario Internacional. Cuba: Gaceta; 1982 [citado 3 Abr 2010]; 38. Disponible en:

http://www.sld.cu/galerias/pdf/sitios/insat/dl-104-1982.pdf

9. Martínez Torres E. Dengue [CD-ROM]. La Habana: Ediciones Digitales; 2003.

10. Organización Mundial de la Salud. Pandemia H1N1 2009 Actualización 99 de la OMS. [Internet]. Ginebra: OMS; 2010 [citado 13 May 2010]. Disponible en:

http://www.who.int/csr/don/2009_09_11/en /index.html

11. De La Osa JA. Control Sanitario Internacional (CSI). Fortalecer el escudo protector del CSI con responsabilidad ciudadana. El Habanero [Internet]. [citado 3 Abr 2010]. Disponible en:

http://www.elhabanero.cubaweb.cu/2009/octubre/nro2686_oct09/cienc_09oct939.ht $\underline{\mathrm{ml}}$

12. Mesa RG, González PJ, Martínez CS, Reyes FM, Fuentes DM, Gómez M L. Experiencias Cubanas. Salud y Desastres. La Habana: Editorial Ciencias Médicas; 2009.

Recibido: 28 de octubre de 2010.

Aprobado: 9 de noviembre de 2010.

Fernando González Isla. Dirección Provincial de Salud. Calle Frexes No. 121 e/ Morales Lemus y Miró CP80100. Holguín, Cuba. Correo electrónico: islafg@dps.hlg.sld.cu 